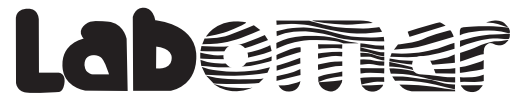

Arquivos de Ciências do Mar

\section{INFLUÊNGIA DAS VARIÁVEIS AMBIENTAIS NA QUALIDADE DA ÁGUA DE UMA LAGOA COSTEIRA TROPICAL NO NORTE DO BRASIL}

\author{
Influence of environmental variables on the water \\ quality of a coastal tropical lagoon in northern Brazil
}

\author{
Maria Eduarda Garcia de Sousa Pereira1, Nils Edvin Asp Neto², \\ Hanna Tereza Garcia de Sousa Moura ${ }^{3}$, Zélia Maria Pimentel Nunes ${ }^{4}$ \\ ${ }^{1}$ Mestre em Biologia Ambiental, Universidade Federal do Pará, Campus Bragança. E-mail: eduardapesca@yahoo.com.br \\ ${ }_{2}^{2}$ Professor do Instituto de Estudos Costeiros, Universidade Federal do Pará, Campus Bragança. E-mail: nilsasp@ufpa.br \\ ${ }^{3}$ Mestranda em Biologia Ambiental, Universidade Federal do Pará, Campus Bragança. E-mail: hannapesca21@yahoo.com.br \\ ${ }^{4}$ Professora do Instituto de Estudos Costeiros, Universidade Federal do Pará, Campus Bragança. E-mail: znunes@yufpa.br
}

\section{RESUMO}

As lagoas costeiras exercem funções importantes de natureza ecológica e socioeconômica, devido à sua alta produtividade e ao uso múltiplo de suas águas. O objetivo desta pesquisa foi avaliar a variação temporal de algumas variáveis ambientais da água da Lagoa Salina (nordeste do Pará), cuja dinâmica apresenta características peculiares, variando em função da sazonalidade, das grandes marés de sizígia e das substâncias húmicas oriundas do manguezal, predominante na bacia de drenagem. A água foi monitorada, mensalmente, durante doze meses, com base nos valores de temperatura, salinidade, oxigênio dissolvido, $\mathrm{pH}$, condutividade elétrica, turbidez, transparência, profundidade e pluviosidade. Os resultados mostraram que somente as variáveis, salinidade, condutividade e $\mathrm{pH}$, apresentaram diferença significativa entre os períodos chuvoso e seco, indicando a influência da pluviosidade. A Análise de Componentes Principais explicou 71,9\% da variação dos dados, com o Componente 1 refletindo a influência da sazonalidade sobre transparência da água, condutividade e pluviosidade, e o Componente 2 refletindo a influência das marés de sizígia e do início do período chuvoso sobre $\mathrm{pH}$ e turbidez. Todas essas características estabelecem, em determinados períodos, condições extremas à biota aquática.

Palavras-chaves: lagoa tropical, sazonalidade, maré de sizígia.

\section{ABSTRACT}

The coastal lagoons play important ecological and socio-economic roles due of their high productivity and multiple uses of the water. This research aimed to evaluate the time variation of a

Recebido: 7 nov 2016

Aceito: 28 nov 2016

Publicado online: 31 mai 2017 
few environmental variables of the Salina Lagoon (northeastern Pará State), whose dynamics display peculiar characteristics as to seasonality of outstanding spring tides, and humic substances derived from the predominant mangrove forest in its draining basin. The water was monitored monthly over a year's period for twelve months, based on the estimates of temperature, salinity, dissolved oxygen, $p H$, conductivity, turbidity, transparency, depth and rainfall. Their results showed that only salinity, conductivity and $p H$ values showed significant differences between the rainy and dry seasons, highlighting the influence of rainfall on the lagoon. The Principal Component Analysis has explained $71.9 \%$ of the data variation, with Component 1 underscoring the influence of seasonality on water transparency, conductivity and rainfall, and Component 2 underscoring the influence of spring tides and early rainy season on $\mathrm{pH}$ and turbidity. All those features can impose, in certain periods, extreme conditions to the aquatic biota.

Keywords: coastal lagoon, seasonality, spring tide.

\section{INTRODUÇÃO}

As Lagoas costeiras são encontradas ao longo do litoral brasileiro, com tamanho variando desde pequenas depressões, preenchidas com águas fluviais, subterrâneas ou marinhas, até grandes sistemas como a Lagoa dos Patos, no Rio Grande do Sul. Destacam-se por suas funções de caráter ecológico e socioeconômico, por abrigarem formas juvenis de peixes e crustáceos que constituem o sustento de populações tradicionais (Furtado \& Pimentel, 2010). Dependendo da sua natureza, podem estar ou não ligadas diretamente ao mar (Knoppers \& Kjerfve, 1999) e, portanto, são consideradas zonas críticas de transição entre a água doce e marinha, altamente heterogênea e dinâmica (Pérez-Ruzafa et al., 2013).

Assim, o estudo da dinâmica desses corpos hídricos permite avaliar o seu funcionamento, bem como o dos organismos que ali se desenvolvem. As características abióticas e bióticas de uma lagoa salina estão relacionadas a condições extremas de fatores climatológicos, como temperatura elevada, baixa umidade relativa do ar, baixos índices pluviométricos e altos níveis de evaporação (Baha AL-deen, 1974), as quais favorecem a colonização por formas jovens com grande capacidade osmorreguladora, passando a condição de berçários para diversas espécies de peixes (Pereira et al., 2015).

A heterogeneidade e a dinâmica desses ecossistemas dificultam a diferenciação das mudanças naturais das induzidas pelo homem (Elliott \& Quintino, 2007) como, por exemplo, eutrofização, alterações dos fluxos de entrada, fragmentação da paisagem e modificação geomorfológica (Stora \& Arnoux, 1983; De Jonge \& De Jong, 2002).

Com base no exposto, o objetivo deste estudo foi avaliar o comportamento de algumas variáveis ambientais da água da lagoa Salina (nordeste do Pará) e seus afluentes, considerando a sazonalidade da região, uma vez que a qualidade da água influencia diretamente na composição da biota estabelecida nesse ecossistema.

\section{MATERIAL E MÉTODOS}

A presente pesquisa foi realizada na Lagoa Salina (Figura 1), inserida na planície costeira bragantina, e formada a partir da construção da estrada que causou a secção de canais de maré e passou a funcionar como barragem (Souza-Filho, 2000). Apesar de situada 
nas adjacências do estuário do Rio Caeté, esse biótopo não apresenta conexão constante com outros ambientes aquáticos adjacentes, exceto durante os períodos de marés equinociais de sizígia. Possui uma área de $0,19 \mathrm{~km} 2$, cercada por bosque de mangue, com profundidade inferior a 1,5 m e fundo lodoso, constituindo-se em área de pesca para as comunidades adjacentes (Goch et al., 2005).

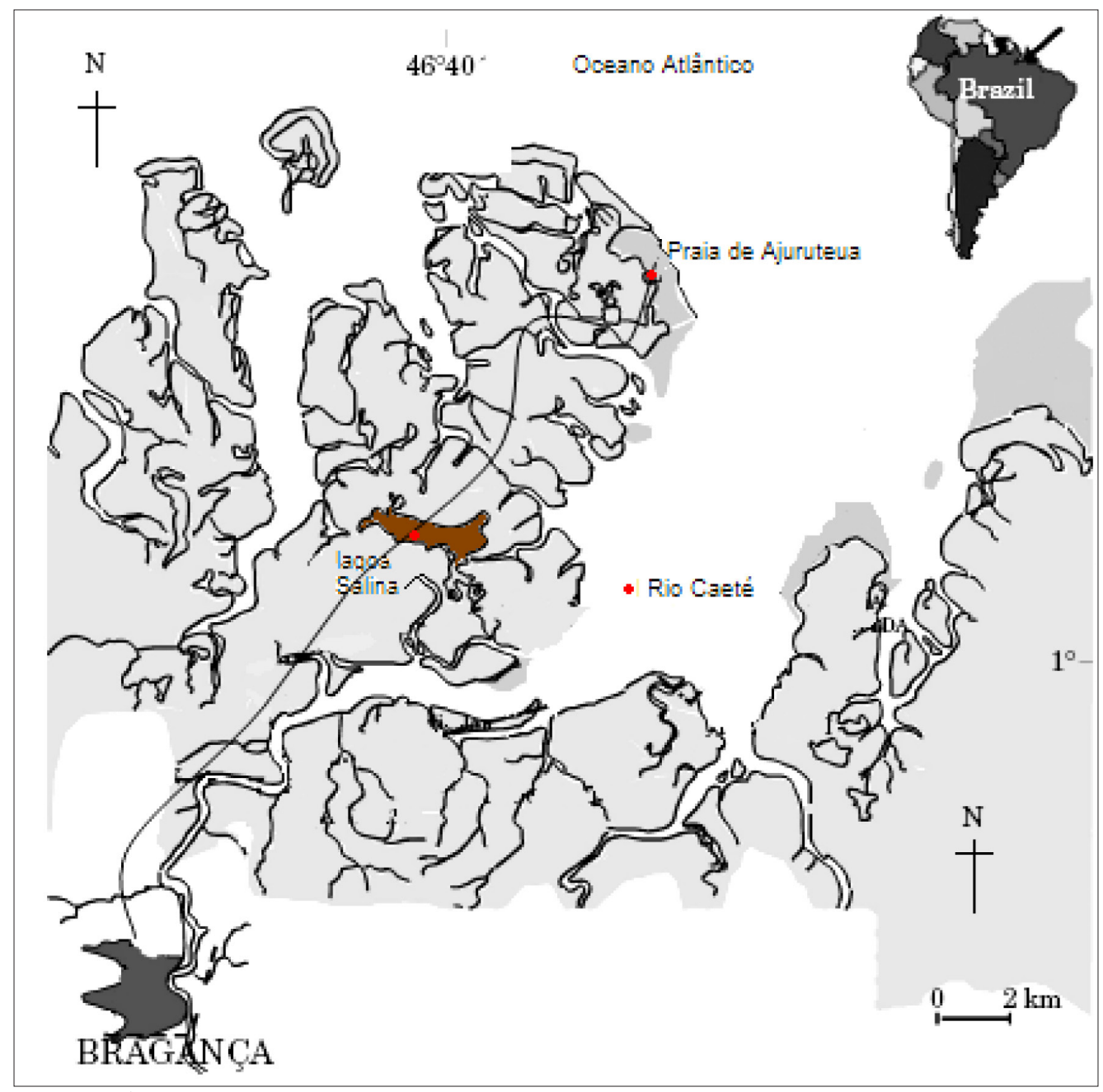

Figura 1 - Área de estudo: Lagoa Salina (Fonte: Barletta et al., 2005).

O clima dessa área pode ser caracterizado como muito úmido, megatérmico, com sazonalidade bem definida e duas estações características: chuvosa, de janeiro a junho e a seca, de julho a dezembro, de acordo com a classificação climática de Köpeen (Souza-Filho \& El-Robrini, 1997). A pluviosidade anual oscila entre 2.500 e 3.000 mm, embora estes valores possam apresentar amplas variações, principalmente quando influenciados por fenômenos atmosféricos de grande escala (Martorano et al., 1993).

O monitoramento mensal da qualidade da água foi realizado entre março/2007 e fevereiro/ 2008, entre as $7 \mathrm{~h}$ e $15 \mathrm{~h}$, com o uso do multianalisador HORIBA U-10, com registro de valores de temperatura $\left({ }^{\circ} \mathrm{C}\right)$, salinidade, oxigênio dissolvido $\left(\mathrm{mg}^{-\mathrm{L}^{-1}}\right), \mathrm{pH}$, condutividade elétrica $\left(\mathrm{mS} . \mathrm{cm}^{-1}\right)$ e turbidez $(\mathrm{UNT})$. A visibilidade de Secchi $(\mathrm{cm})$ e a profundidade do local $(\mathrm{cm})$ foram obtidas com o auxílio do disco de Secchi. A caracterização 
meteorológica da área de estudo foi feita a partir dos valores acumulados mensais de pluviosidade pluviométrica total (mm), fornecidos pela Estação Meteorológica de Tracuateua, Instituto Nacional de Meteorologia - INMET.

As variáveis físicas e químicas foram descritas tendo como base o valor médio ou a mediana, e os valores mínimo e máximo determinados mensalmente. Verificou-se também a relação dessas variáveis em relação à sazonalidade da pluviosidade na região: estação chuvosa (janeiro - junho) e estação seca (julho - dezembro). Inicialmente, os dados foram analisados quanto à normalidade da distribuição e à homogeneidade das variâncias por meio dos testes de Liliefors e Bartlett, respectivamente. Utilizou-se o teste U de MannWhitney para dados heterogêneos ( $\mathrm{pH}$, salinidade e condutividade) e ANOVA para os dados homogêneos (oxigênio dissolvido, temperatura, transparência e turbidez), para um nível crítico de significância de $\alpha=0,05$.

A Análise de Componentes Principais (ACP) foi o método de ordenação utilizado neste trabalho para identificar os principais componentes responsáveis pela variação dos dados ambientais na Lagoa Salina, tendo como base a matriz de correlação. Para a análise dos dados, confecção dos gráficos e tabelas utilizou-se os Softwares STATISTICA versão 7.0, PRIMER 6.0 e Excel 2000.

\section{RESULTADOS}

A pluviosidade mensal durante o período analisado variou de 10,4 $\mathrm{mm}$ (outubro) a

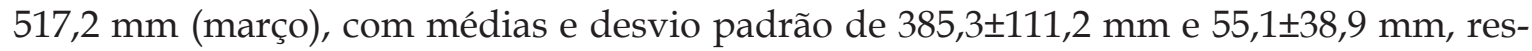
pectivamente no primeiro e segundo semestres de 2007.

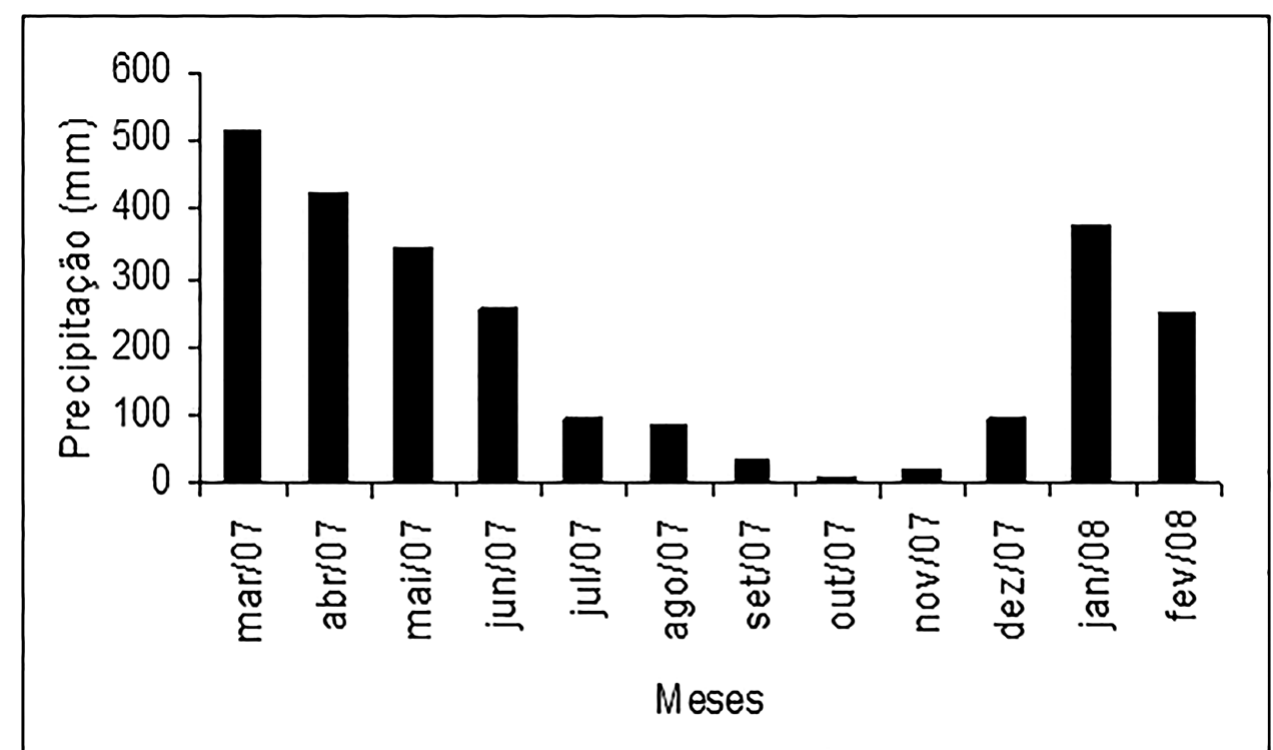

Figura 2 - Pluviosidade mensal registrada na área da Lagoa Salina, nordeste do Pará, no período março/2007 - fevereiro/2008. (Fonte: Estação Meteorológica de Tracuateua, Instituto Nacional de Meteorologia - INMET).

Nos meses de março, abril e junho as variáveis abióticas foram determinadas em um único ponto de coleta (Tabela I), sem repetição, mas os resultados obtidos apresentaram um padrão compatível com o esperado para o período chuvoso. 
Tabela I - Análise pontual da água na Lagoa Salina em três meses de 2007.

\begin{tabular}{l|c|c|c}
\hline \multicolumn{1}{c|}{ Variável } & Março & Abril & Junho \\
\hline Profundidade $(\mathrm{cm})$ & 21 & 20 & 24 \\
Temperatura $\left({ }^{\circ} \mathrm{C}\right)$ & 29,1 & 28,1 & 29,8 \\
Oxigênio $\left(\mathrm{mg}^{-1} \mathrm{~L}^{-1}\right)$ & 4,18 & 4,23 & 5,21 \\
$\mathrm{pH}$ & 6,9 & 7,6 & 8,0 \\
Salinidade & 1,43 & 1,51 & 1,47 \\
Condutividade $\left(\mathrm{mS} . \mathrm{cm}^{-1}\right)$ & 22,4 & 24,7 & 32,2 \\
Transparência $(\mathrm{cm})$ & 21 & 20 & 24 \\
Turbidez $(\mathrm{UNT})$ & 124 & 128 & 137 \\
\hline
\end{tabular}

A profundidade dos locais amostrados na lagoa variou de $20 \mathrm{~cm}$ (abril/2007) a 84,5 cm (fevereiro/2008) (Figura 3), com tendência de aumento ao longo dos meses, fato que está relacionado com o deslocamento das áreas de amostragem para o centro da lagoa em virtude da redução do nível da água.

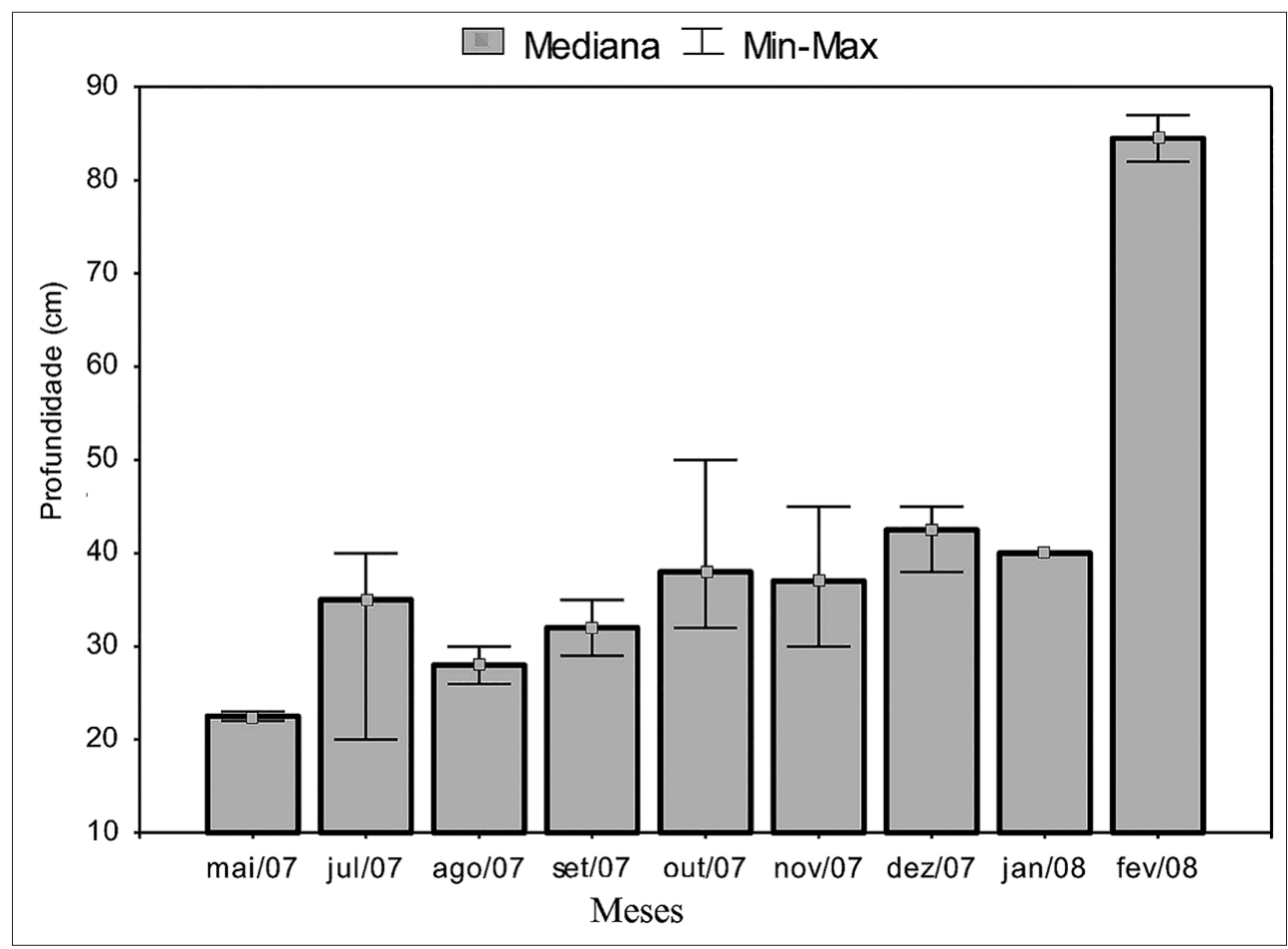

Figura 3 - Profundidade mediana dos locais de amostragem na Lagoa Salina, de maio/2007 a fevereiro/2008.

A visibilidade mediana do disco de Secchi na água variou de $18,5-70,0 \mathrm{~cm}$, sendo a mínima registrada em maio/2007 e a máxima, em fevereiro/2008, com tendência de crescimento gradativo ao longo dos meses nos pontos amostrados. Verificou-se que os menores valores da transparência foram registrados no período chuvoso e os maiores no período seco, com exceção dos meses de janeiro e fevereiro (Figura 4-A). Com base nos valores de transparência registrados não houve diferença significativa $(p>0,05)$ entre os períodos chuvoso e seco (Figura 4-A').

Durante o horário das coletas, a temperatura variou de $27,3^{\circ} \mathrm{C}$ a $32,6^{\circ} \mathrm{C}$, com distribuição dos valores medianos na faixa de $28,0-30,6^{\circ} \mathrm{C}$, nos meses de janeiro e fevereiro, 
respectivamente (Figura 4-B), não sendo observado diferença estatisticamente significativa entre os períodos chuvoso e o seco $(\mathrm{p}>0,05)$ (Figura $\left.4-\mathrm{B}^{\prime}\right)$.

A concentração de oxigênio dissolvido na lagoa durante o horário das coletas variou de 3,52 mg. $\mathrm{L}^{-1}$ a $8,92 \mathrm{mg} . \mathrm{L}^{-1}$. Ficando a distribuição dos valores medianos na faixa de 4,27 mg. $\mathrm{L}^{-1}$ (maio) a 8,11 mg. $\mathrm{L}^{-1}$ (janeiro) (Figura 4-C). Os valores registrados para essa variável apresentou diferença estatisticamente significativa entre os períodos chuvoso e o seco $(\mathrm{p}>0,05)$ (Figura 4-C').

A água da Lagoa Salina apresentou pH neutro com tendência a alcalino, com exceção dos meses de janeiro e fevereiro, quando se registrou $\mathrm{pH}$ ácido. $\mathrm{O}$ valor mediano do $\mathrm{pH}$ variou de 4,8 (janeiro) a 8,76 (setembro), conforme se observa na Figura 4-D. Os valores de $\mathrm{pH}$ durante o período chuvoso, com tendência para acidez, foram significativamente inferiores aos do período seco, com tendência para alcalinidade $(\mathrm{p}<0,05)$ (Figura 4-D').

A salinidade variou de 14, no mês de agosto, a 40 em outubro, novembro e dezembro, observando-se que os menores valores foram registrados no período chuvoso e os maiores no período seco. Verificou-se relação inversa dessa variável com a pluviometria, com exceção de maio, mês em que esta não ocorreu devido à influência das grandes marés de sizígia, que introduzem águas de origem marinha na lagoa (Figura 4-E). A salinidade do período chuvoso foi significativamente inferior $(\mathrm{p}<0,05)$ à do período seco (Figura $4-\mathrm{E}^{\prime}$ ).

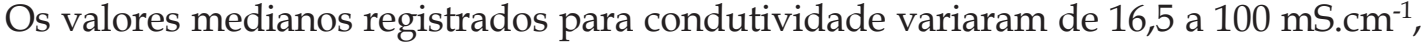
sendo o menor obtido em agosto e o maior, em dezembro. Observa-se que os menores valores da condutividade foram registrados no período chuvoso e os maiores no período seco, com diferença estatisticamente significante $(p<0,05)$ entre as duas estações (Figura 4 - F/F').

Os valores medianos de turbidez variaram de 33,2 NTU a 166,4 NTU em janeiro e setembro, respectivamente, em registro de diferença significativa $(p>0,05)$ desta variável entre os períodos chuvoso e o seco (Figura 4 - G/G').

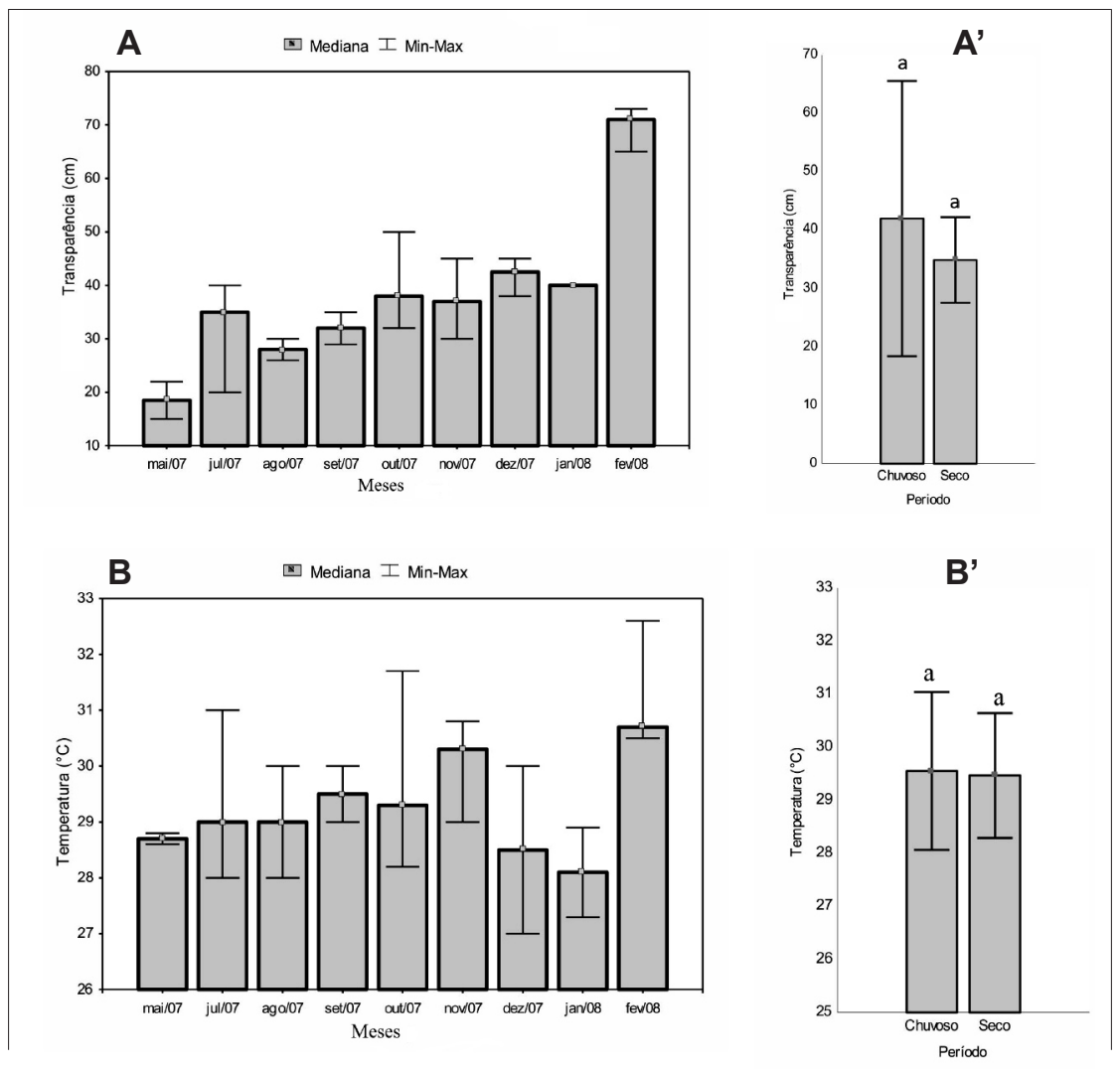



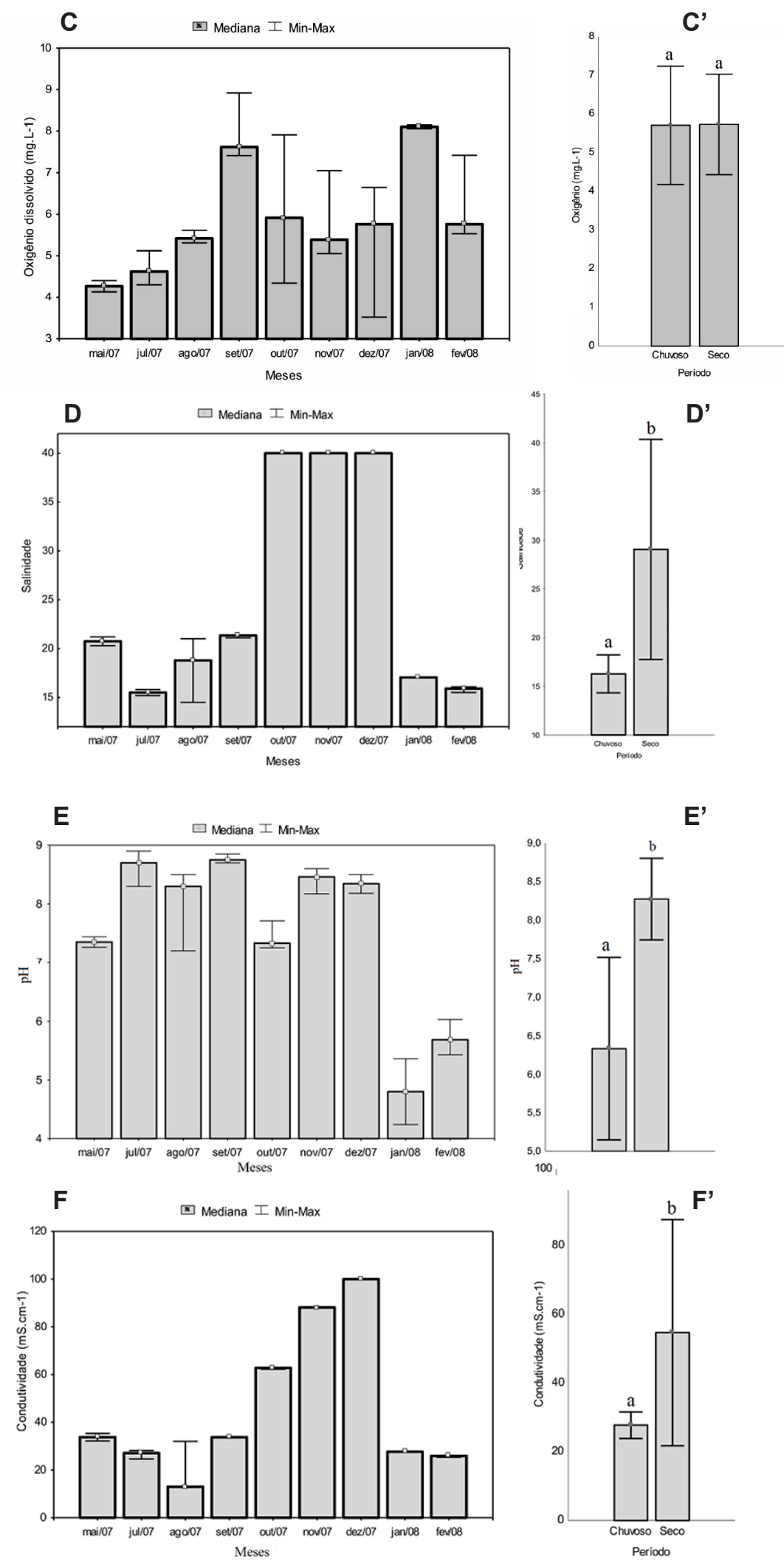

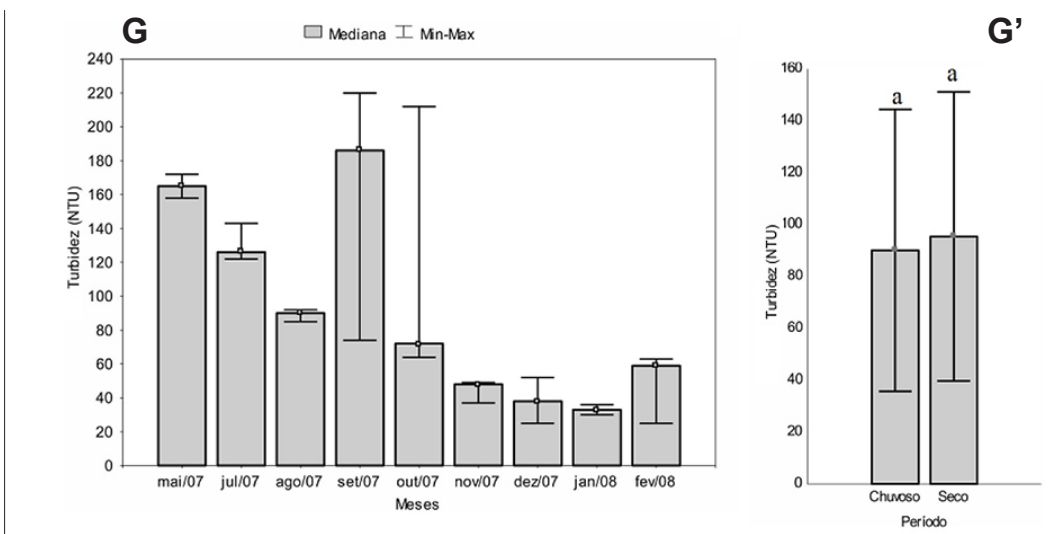

Figura 4 - Valores medianos da transparência (A), temperatura (B), oxigênio (C), pH (D), salinidade (E), condutividade (F) e turbidez (G) da água da lagoa Salina, de maio/2007 a fevereiro/2008; Valores médios ( \pm D.P.) da transparência $\left(A^{\prime}\right)$, temperatura $\left(B^{\prime}\right)$, oxigênio $\left(C^{\prime}\right)$, $\mathrm{pH}\left(\mathrm{D}^{\prime}\right)$, salinidade $\left(\mathrm{E}^{\prime}\right)$, condutividade $\left(\mathrm{F}^{\prime}\right)$ e turbidez $\left(\mathrm{G}^{\prime}\right)$. Letras diferentes acima das barras indicam diferença estatisticamente significante $(p<0,05)$ entre os períodos chuvoso e seco.

A partir da Análise de Componentes Principais (ACP) realizada com as variáveis ambientais, observou-se que os dois primeiros componentes explicaram 71,9\% da variabilidade total dos dados. O Componente $1(40,4 \%)$ esteve relacionado positivamente com a transparência e a condutividade e negativamente à turbidez e à pluviosidade, refletindo a influência da sazonalidade sobre as variáveis ambientais. Observou-se que nos meses de outubro, novembro e dezembro a água da lagoa encontrava-se mais salina e transparente em decorrência da diminuição da pluviosidade e o aumento da evaporação. Nos meses de março, abril, maio e junho, observou-se o processo inverso pelo efeito redutor do aumento da pluviosidade sobre a condutividade, carreando material particulado em suspensão para lagoa e diminuindo sua transparência. Os meses de julho e agosto, apesar de considerados como período seco, ainda foram influenciados pela pluviosidade em decorrência da drenagem das áreas adjacentes (Figura 5).

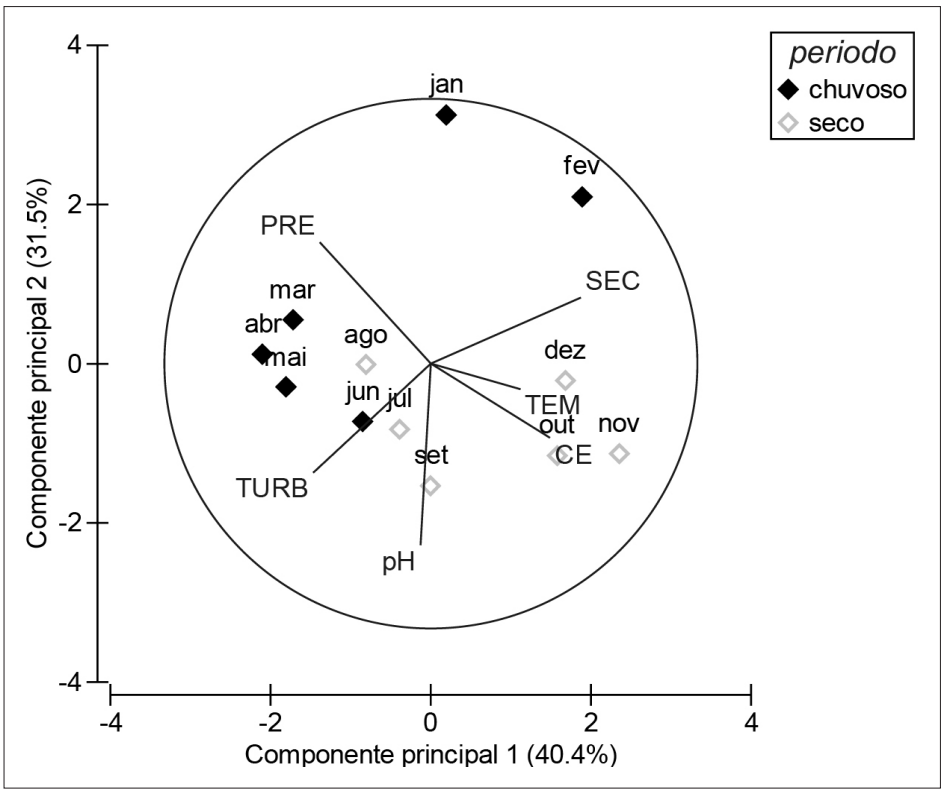

Figura 5 - Círculo de correlação das variáveis ambientais na Lagoa Salina $(\mathrm{SEC}=$ Transparência, $\mathrm{TEM}=$ Temperatura, $\mathrm{CE}=$ Condutividade, PRE $=$ Precipitação e TURB $=$ Turbidez). 
O Componente $2(31,5 \%)$ esteve relacionado negativamente ao $\mathrm{pH}$ e à turbidez, o qual refletiu as condições registradas no início do período chuvoso, quando a chuva promove o escoamento superficial do manguezal adjacente. Este é responsável pelo carreamento de ácidos húmicos e fúlvicos para a lagoa, deixando a água ácida e favorecendo a adsorção e a sedimentação das partículas em suspensão, consequentemente, diminuindo sua turbidez. Essas condições foram observadas nos meses de janeiro e fevereiro, diferente de setembro, quando a água se encontrava alcalina e turva, em decorrência das grandes marés de sizígia (Figura 5).

De acordo com a ACP, o pH apresentou correlação positiva com a turbidez $(\mathrm{r}=0,456)$, e negativa com a pluviosidade $(r=-0,566)$ e a transparência $(r=-0,419)$. Essas condições de $\mathrm{pH}$ ácido, baixa turbidez, índices elevados de pluviosidade e aumento na visibilidade do disco de Secchi ocorreram no início do período chuvoso de 2008 (Tabela II).

A pluviosidade correlacionou-se negativamente à condutividade $(r=-0,527)$. A chuva promoveu a diluição dos sais contidos na água, reduzindo, portanto, a condutividade. A correlação entre a condutividade e a turbidez foi negativa $(r=-0,470)$, com os maiores valores de turbidez sendo registrados no período chuvoso, ao contrário da condutividade.

A temperatura apresentou correlação direta com a transparência da água $(r=0,607)$. Águas com uma maior visibilidade do disco de Secchi promoveram a penetração da radiação solar na coluna d'água aumentando, assim, a temperatura, condição observada principalmente durante o período seco e na transição do seco/chuvoso. A turbidez apresentou correlação negativa com a transparência $(r=-0,662)$, pois os sólidos em suspensão causam dispersão da radiação solar subaquática.

Tabela II - Matriz de correlação entre as variáveis da Análise de Componentes Principais.

\begin{tabular}{|c|c|c|c|c|c|c|}
\hline Variáveis & $\mathrm{pH}$ & Pluviosidade & Temperatura & Condutividade & Turbidez & Transparência \\
\hline $\mathrm{pH}$ & 1,000 & & & & & \\
\hline Pluviosidade & $-0,566$ & 1,000 & & & & \\
\hline Temperatura & 0,058 & $-0,346$ & 1,000 & & & \\
\hline Condutividade & 0,336 & $-0,527$ & 0,041 & 1,000 & & \\
\hline Turbidez & 0,456 & 0,140 & $-0,069$ & $-0,470$ & 1,000 & \\
\hline Transparência & $-0,419$ & $-0,303$ & 0,607 & 0,234 & $-0,662$ & 1,000 \\
\hline
\end{tabular}

\section{DISCUSSÃO}

As lagoas costeiras são ecossistemas com características particulares e apresentam diferentes interesses que podem gerar conflito, a exemplo da pesca, turismo e aquicultura, atividades que funcionam como vetores do desenvolvimento socioeconômico, de modo que é fundamental o conhecimento de suas características (Pérez-Ruzafa et al., 2011).

Na Lagoa Salina, as variações físico-químicas da água ocorrem, principalmente, em decorrência dos índices de pluviosidade e da ocorrência das marés equinociais de sizígias, uma vez que existe ausência de uma comunicação constante com o mar ou com o estuário. Na região de estudo, a variação mensal da pluviosidade caracterizou a sazonalidade, com duas estações marcantes, a chuvosa, de janeiro a junho, e a seca, de julho a dezembro, já investigada em trabalho realizado por Souza-Filho \& El-Robrini (1997). Esta situação promove a mistura de água salgada e doce determinando as propriedades físicas e químicas da água, os quais variam gradativamente e entre os ciclos anuais, devido à imprevisibili- 
dade do fluxo de entrada de água nesse ecossistema. Essa entrada de água vem ao longo do tempo sendo alterada devido à ação antrópica (Smith, 2003; Elliott \& Quintino, 2007; Viaroli et al., 2007).

Na lagoa salina a pluviosidade influenciou diretamente na salinidade, na condutividade e no valor do $\mathrm{pH}$, funcionando como um regulador na variação das variáveis físicas e químicas da água, bem como na estrutura temporal e espacial de peixes (Paperno \& Brodie, 2004).

A água dessa lagoa variou de oligosalina a hipersalina, de acordo com a classificação sugerida no Symposium of Brackish Waters, de 1958 (The Venice System), atribuídas principalmente às diferentes taxas anuais de pluviosidade e evaporação. A salinidade é considerada um fator limitante da ictiofauna da Lagoa Salina (Goch et al., 2005) e, dependendo da influência das águas doce ou marinha, a composição das espécies é alternada e estratégias reprodutivas diferenciadas são adotadas (Pérez-Ruzafa et al., 2013).

Nesta pesquisa, os valores de condutividade foram menores no período chuvoso e maiores no período seco, exceto em agosto, mês em que se observou a influência da pluviosidade, através da drenagem de águas adjacentes. Valores superiores ao desta pesquisa foram observados na Lagoa Jacuném, em decorrência do aporte de íons provenientes da bacia de drenagem. Esses resultados demonstram que os valores de condutividade em ecossistemas localizados em regiões tropicais estão mais relacionados às características geoquímicas locais e às condições climáticas (período chuvoso e seco) do que ao estado trófico do ambiente (Esteves et al., 1988).

No presente estudo, atribuiu-se a acidez da água ao início do período chuvoso, quando o escoamento superficial do manguezal adjacente carreia substâncias húmicas para a lagoa, contribuindo para a acidez da água. Ide et al. (2014), observaram a variação do $\mathrm{pH}$, em função dos ventos, ao fenômeno de diluição do sistema nos períodos de águas altas, à biomassa fitoplanctônica, aos processos de decomposição e à presença de substâncias húmicas

A temperatura da água pode ser influenciada pelas condições climáticas locais, como pluviosidade, temperatura do ar, radiação solar, umidade e velocidade do vento (Stefan \& Preud'Homme, 1993) e, em lagoas tropicais é superior a $25^{\circ} \mathrm{C}$ durante a maior parte do ano (Esteves et al., 1988), como se observou neste estudo com variação na faixa de $28-30,6^{\circ} \mathrm{C}$ e pequena divergência sazonal.

Os valores de oxigênio foram superiores a 3,5 mg. $\mathrm{L}^{-1}$, porém inferiores ao registrado por Goch et al (2005) de 5,8 mg. $\mathrm{L}^{-1}$ ao realizar estudos na Lagoa Salina ao longo do ano. Apesar do valor encontrado no presente estudo ser inferior ao registrado anteriormente, essa variável não foi um fator limitante à ictiofauna, pois se manteve acima de $2 \mathrm{mg}$. $\mathrm{L}^{-1}$.

A transparência do disco de Secchi variou de $18,5 \mathrm{~cm}$ a $70 \mathrm{~cm}$ na Lagoa Salina, acompanhando os valores da profundidade nos locais amostrados, que atinge um valor máximo de $150 \mathrm{~cm}$ (Goch et al., 2005), o que favorece a penetração da radiação solar. A hidrodinâmica está intimamente condicionada pela topografia do fundo e o vento, afetando a coluna d'água, promovendo a ressuspensão de materiais, nutrientes e organismos pequenos a partir da camada superficial do sedimento (Pérez-Ruzafa et al., 2013).

Os valores de turbidez variaram de 33,2 a 166,4 NTU em janeiro e setembro, respectivamente, tendo o menor valor registrado coincidido com o início do período chuvoso, quando acredita-se que a chuva promoveu a entrada de substâncias húmicas na lagoa, diminuindo o pH, promovendo a adsorção e sedimentação das partículas em suspensão. Os compostos húmicos coloidais oferecem uma área superficial grande, apropriada para a adsorção 
de matéria orgânica e inorgânica (Poirrier et al., 1972). No período chuvoso, aumenta a entrada de matéria orgânica oriunda do manguezal nos ecossistemas aquáticos adjacentes.

Estudos realizados em outras lagoas indicam que o fator sazonalidade é de extrema importância na distribuição das variáveis físicas e químicas (Ishitobi et al., 2000; VegaCendejas e Santillana, 2004). Martins et al. (2006) observaram que a sazonalidade, também, exerce um papel importante na composição e na abundância das diferentes espécies zooplanctônicas da Lagoa Salina.

O Componente 2 influenciou negativamente o $\mathrm{pH}$ e positivamente a pluviosidade, refletindo as condições do início do período chuvoso (janeiro e fevereiro), quando a pluviosidade promoveu o escoamento superficial do manguezal adjacente, carreando provavelmente substâncias húmicas para a lagoa, diminuindo a turbidez e promovendo a acidez da água. Por outro lado, esse componente também refletiu a influência das grandes marés de sizígia. No mês de setembro quando ocorrem as grandes marés, a influência da água do mar contribuiu para o aumento do $\mathrm{pH}$ e elevação da turbidez. Os carbonatos presentes nos sprays marinhos que chegam à lagoa são responsáveis pelos altos valores de pH (Simonassi, 2001).

\section{CONCLUSÃO}

A dinâmica das variáveis ambientais da água da Lagoa Salina, no nordeste do Pará, apresentou características peculiares, variando em função, principalmente, da sazonalidade e das grandes marés de sizígia. Essas características estabelecem, em determinados períodos, condições extremas à biota aquática, favorecendo o estabelecimento de espécies com plasticidade ecológica.

\section{REFERÊNCIAS BIBLIOGRÁFICAS}

Baha Al-Deen, B. Fatores que deve reunir uma region para a construccion de uma salina por evaporacion solar del água de mar y aspectos generales de este tipo de salinas. Lagena, v.34, p.9-11, 1974.

Barletta, M.; Barletta-Bergan, A.; Saint-Paul, U.; Hubold, G. The role of salinity in structuring the fish assemblages in a tropical estuary. J. Fish Biol., v.66, p.45-72, 2005.

De Jonge, V.N. \& De Jong, D.J. Ecological restoration in coastal areas in the Netherlands: concepts, dilemmas and some examples. Hydrobiologia, v.478, p.7-28, 2002.

Elliott, M. \& Quintino, V. The estuarine quality paradox, environmental homeostasis and the difficulty of detecting anthropogenic stress in naturally stressed areas. Mar. Pollut. Bull., v.54, p.640-645, 2007.

Esteves, F.A.; Bozelli, R.L.; Camargo, A.F.M.; Roland, F. \& Thomaz, S.M. Variação diária (24 horas) de temperatura, $\mathrm{O}_{2}$ dissolvido, $\mathrm{pH}$ e alcalinidade em duas lagoas costeiras do Estado do Rio de Janeiro e suas implicações no metabolismo destes ecossistemas. Acta Limnol. Brasil., v.2, p.99-127, 1988.

Furtado, A.M.M. \& Pimentel, M.A.S. Degradação ambiental e cultural decorrente da atividade turística na zona costeira do nordeste paraense. Estudo de caso no município de Curuçá-PA, p.1-12, in VI Seminário Latino Americano de Geografia Física, Coimbra, 2010.

Goch, Y.G.F.; Krumme, U.; Saint-Paul, U. \& Zuanon, J.A.S. Seasonal and diurnal changes 
in the fish fauna composition of a mangrove lake in the Caeté Estuary, North Brazil. Amazoniana, v.18, n.3/4, p.299-315, 2005.

Ide, A.H.; Cardoso, F.D.; Santos, M.M.; Azevedo, J.C.R. Variação sazonal de algumas características limnológicas da lagoa dos Patos/MS. SaBios: Rev. Saúde e Biol., v.9, n.1, p.21-33, 2014.

Ishitobi, Y.; Hiratsuka, J. \& Kuwabara, H. Comparison of fish fauna in three areas of adjacent eutrophic estuarine lagoons with different salinity. J. Mar. System., v.26, p.171-181, 2000.

Knoppers, B. \& Kjerfve, B. Coastal lagoons of southeastern Brazil: Physical and biogeochemical characteristics, p.35-66, in Perillo, G.M.E, Piccolo, M.C. \& Quivira, M.P. (eds,), Estuaries of South America. Springer, Berlin, 1999.

Martins, A.A.V. \& Costa, R.A.M. \& Pereira, L.C.C. Distribuição espaço-temporal da comunidade zooplanctônica de uma lagoa costeira artificial na região amazônica, Bragança, Pará, Brasil. Bol. Mus. Emílio Goeldi; ser. Ciên. Nat., v.2, n.3, p.93-101, 2006.

Martorano, L.G.; Perreira, L.C.; Cézar, E.G.M. \& Pereira, I.C.B. Estudos climáticos do Estado do Pará, classificação climática (Koppen) e deficiência hídrica (Thornthwhite, Mather). SUDAM/ EMBRAPA, 53 p., Belém, 1993.

Paperno, R. \& Brodie, R.B. Effects of environmental variables upon the spatial and temporal structure of a fish community in a small, freshwater tributary of the Indian River Lagoon, Florida. Est. Coast. Shelf Sci., v.61, p.229-241, 2004.

Pereira, M.E.G.S; Silva, B.B.; Rocha, R.M.; Asp-Neto, N.E.; Silva, C.S.; Nunes, Z.M.P. Bioecologia do robalo-flexa, Centropomus undecimalis, em lagoa costeira tropical no norte do Brasil. Bol. Inst. Pesca, v.41, n.3, p.457-469, 2015.

Pérez-Ruzafa, A.; Marcos, C.; Pérez-Ruzafa, I.M. \& Pérez-Marcos, M. Coastal lagoons: "transitional ecosystems" between transitional and coastal waters. J. Coast. Conserv., v.15, p.369-392, 2011.

Pérez-Ruzafa, A.; Marcos, C.; Pérez-Ruzafa, I.M. \& Pérez-Marcos, M. Are coastal lagoons physically or biologically controlled ecosystems? Revisiting $r$ vs. $k$ strategies in coastal lagoons and estuaries. Est. Coast. Shelf Sci., v.132, p.17-33, 2013.

Poirrier, M.A.; Bordelon, B.R \& Laseter, J.L. Adsorption and concentration of dissolved carbono-14 DDT by coloring colloids in surface waters. Environ: Sci. Technol., v.6, p.10331035, 1972.

Simonassi, J.C. Caracterização da Lagoa do Peri, através da análise de parâmetros físicos-químicos e biológicos, como subsídio ao gerenciamento dos recursos hídricos da Ilha de Santa Catarina, SC, Brasil. Dissertação de Mestrado, Programa de Pós-graduação em Engenharia de Produção, Universidade Federal de Santa Catarina, 72 p., Florianópolis, 2001.

Smith, V.H. Eutrophication of freshwater and coastal marine ecosystems: a global problem. Environ. Sci. Pollut. Res., v.10, 126-139, 2003.

Souza-filho, P.W.M. \& El-Robrini, M. A influência das variações do nível do mar na sedimentação da Planície Costeira Bragantina durante o Holoceno - nordeste do Pará, Brasil, p.307-337, in Costa, M.L. \& Angélica, R.S. (eds.), Contribuições à geologia da Amazônia. FINEP/ SBG, Belém, 1997.

Souza-Filho, P.W.M. \& El-Robrini, M. Geomorphology of the Bragança coastal zone, northeastern Pará State. Rev. Brasil. Geociên., v.30, p.518-522, 2000. 
Stefan, H.G. \& Predu'Homme, E.B. Stream temperature estimation from air temperature. J. Am. Water Resour. Assoc., v.29, n.1, p.27-45, 1993.

Stora, G. \& Arnoux, A. Effects of large freshwater diversions on benthos of a Mediterranean lagoon. Estuaries, v.2, p.115-125, 1983.

Vega-Cendejas, M.E. \& Santillana, M.H. Fish community structure and dynamics in a coastal hypersaline lagoon: rio Lagartos, Yucatan, Mexico. Est. Coast. Shell. Sci., v. 60, p.285299, 2004.

Viaroli, P.; Lasserre, P. \& Campostrini, P. Lagoons and coastal wetlands: preface. Hydrobiologia, v.577, p.1-3, 2007. 